\title{
The human endosalpinx: anatomical three-dimensional study and reconstruction using confocal microtomography
}

\author{
Pedro Teixeira Castro ${ }^{1,2 B, D, F}$, Osvaldo Luiz Aranda ${ }^{1,3 B, D}$, Ana Paula Pinho Matos ${ }^{2 B, F}$, Edson Marchiori ${ }^{10}$, \\ Luiz Felipe Bittencourt de Araújo ${ }^{4 D}$, Haimon Diniz Lopes Alves ${ }^{5,6 A, D}$, Alessandra Silveira Machado ${ }^{6 \mathrm{E}}$, \\ Ricardo Tadeu Lopes ${ }^{6 A, E}$, Heron Werner ${ }^{2 A, D, F}$, Edward Araujo Júnior ${ }^{7 E}$ \\ 'Department of Radiology, Federal University of Rio de Janeiro (UFRJ), Rio de Janeiro-RJ, Brazil \\ 2Department of Radiology, Clínica Diagnóstico por Imagem (CDPI), Rio de Janeiro-RJ, Brazil \\ ${ }^{3}$ Department of Obstetrics and Gynecology, Vassouras University, Vassouras-RJ Brazil \\ ${ }^{4}$ Department of Maternal and Child, Fluminense Federal University (UFF), Niterói-RJ, Brazil \\ ${ }^{5}$ Applied Physics and Thermodynamics Department, Physics Institute, State University of Rio de Janeiro (UERJ), Rio de Janeiro-RJ, Brazil \\ ${ }^{6}$ Nuclear Engineering Program (PEN/COPPE), Federal University of Rio de Janeiro (UFRJ), Rio de Janeiro-RJ, Brazil \\ ${ }^{7}$ Department of Obstetrics, Paulista School of Medicine - Federal University of São Paulo (EPM-UNIFESP), São Paulo-SP, Brazil
}

\section{Abstract}

Purpose: To evaluate in three dimensions (3D) the human endosalpinx and reconstruct its surface along its different anatomical segments, without the injection or insertion of luminal contrasts, using confocal microtomography (micro-CT).

Material and methods: 15 fallopian tubes (FT) from 14 women in reproductive age from procedures for benign disease or sterilization were selected. The specimens were fixed in formalin and stained with Lugol solution. Micro-CT studies were conducted on the specimens using protocols adapted from biological studies, to acquire images to reconstruct in $3 \mathrm{D}$ the endosalpinx surface.

Results: From these specimens, 6 presented the intra-mural segment, 14 presented the isthmus and 15 presented the ampulla and fimbria segment of the FT. The specimen presented tissue definition, and contrast sufficient for FT endosalpinx morphological analysis and lumen definition. The intramural portion presented initially a mucosal projection toward the lumen, bending on its own axis, and increased numbers of projections towards the isthmic portion, where the projections become longer more numerous. The endosalpinx becomes more tortuous, the lumen diameter increases and the mucosal projections become more bulky in the ampullary portion, with the projections less present on the antimesenteric side. The infundibular portion is marked with the organized and predictable endosalpinx, the abdominal ostium is cleared demonstrated, with the reduction of the endosalpinx volume. The fimbria demonstrated a small relation between fringes and intratubal endosalpinx.

Conclusions: Microscopic anatomy of different segments of the human FT mucosa can be analyzed and reconstructed in $3 \mathrm{D}$ with histological correlation using micro-CT.

Key words: fallopian tubes, anatomy, three-dimensional imaging, multislice computed tomography.

\section{Correspondence address:}

Prof. Edward Araujo Júnior, PhD, Rua Belchior de Azevedo, 156 apto. 111 Torre Vitoria, Department of Obstetrics, Paulista School of Medicine - Federal University

of São Paulo (EPM-UNIFESP), São Paulo-SP, Brazil, CEP 05089-030, phone/fax: +55-11-37965944, e-mail: araujojred@terra.com.br

Authors' contribution:

A Study design · B Data collection · C Statistical analysis · D Data interpretation · E Manuscript preparation · F Literature search · G Funds collection 


\section{Introduction}

Reproduction is a complex and multifactorial process that allows the perpetuation of species. The fallopian tube (FT) has a vital role in mammalian reproduction. In addition to linking the gonads to the uterine cavity, the FT has morphological and molecular characteristics that allow the occurrence of important reproductive processes, including the maturation, storage, and transport of gametes; fertilization; fluid transport; and embryonic development [1].

The human FT was described for the first time in Hindu texts 3,000 years ago and was initially correlated with reproductive function by Galen circa 200 AD. In 1543, Andrea Vesalius published De Humani Corporis Fabrica, describing the FT as a trump-shaped duct and compared the FT and ovary to the male ducts and testes. The ovaries (female testes) were rounded by the "semen-conveying" vessels, in an era when the uterus was regarded as the origin of the embryo, following Galen's perspective. In 1561, Gabrielle Fallopius wrote the most accurate description of the oviduct in his Observationes anatomicae, which bears his name [2].

The role of the FT in sperm transport was suggested by de Graaf in 1672 when observing the follicles (then designated "eggs"), which disappeared after copulation, and the appearance of blastocysts in the uterine cavity of rabbits a few days later. Since then, the FT has been considered to be essential in human reproduction. Many reproductive events occur in the FT lumen, where conditions are favorable for fertilization. Moreover, the FT lumen is filled with tubal fluid and is surrounded by the endosalpinx [2].

The endosalpinx has been described anatomically and in three dimensions (3D) in several mammals, including pigs, cows, pig-tailed monkeys, and sheep [3-6]. In the human FT, the endosalpinx has been graphically represented using radiological techniques in studies that evaluated FT patency more than 100 years back [7]. In 1914, Cary studied the FT lumen by X-ray using colloidal silver (Collargol) as the contrast media. The endosalpinx has also been studied using hysterosonography [8], hysterosalpingography, and, more recently, three-dimensional reconstructions of the tubal lumen by magnetic resonance imaging (MRI) [9]. However, these methods use low resolution images or contrast media that are injected under pressure to distend the uterine cavity, causing the dilation and distortion of the FT lumen.

The understanding of physiological processes depends on the knowledge of the anatomy of the FT in healthy as well as pathological conditions [10]. This knowledge is even more important in microscopic anatomy because traditional microscopy has limitations, such as distortions, and three-dimensional reconstruction methods usually destroy the samples, thereby limiting the analysis of human specimens [11].
Recently, microfocus computed tomography (microCT) was shown to be useful for evaluating the microscopic anatomy of biological samples. With the use of $\mathrm{X}$-rays, micro-CT makes three-dimensional reconstructions of biological tissues on a microscopic scale [12]. Biological tissues can be evaluated using micro-CT after contrast uptake, and their anatomical characteristics are preserved for later analysis using traditional microscopic methods, thereby improving the study of human tissues.

The objective of this study is to evaluate the morphology of the human endosalpinx as well as its relationship with the FT segments using micro-CT without the need to inject contrast media in the FT lumen.

\section{Material and methods}

This study is part of a study involving the role of micro-CT in the study of human tissues, with special attention to gynecological conditions. The study was approved by the Local Ethics Committee (\#56031916.0.0000.5290). In a prospective selection of cases, patients scheduled to undergo surgical procedures for benign gynecological conditions (including adenomyosis, fibroids and surgical sterilization) in the University Hospital agreed to participate in the study and provided written informed consent for the use of their tissues for research purposes. Patients with suspected malignant conditions were not included. Patients with macroscopically normal FTs were included in this study. The surgical removal of the FT complied with standard surgical techniques.

\section{Specimen preparation}

Fifteen FT specimens were selected. Twelve specimen were fixed in $10 \%$ formalin solution for $>24 \mathrm{~h}$ at room temperature for preservation of the tissue and cellular architecture [13]. The specimens were washed twice with distilled water. Iodine staining was performed to enhance tissue contrast using 10\% Lugol diluted in physiological solution for $72 \mathrm{~h}$ [14]. Three specimens were immersed in a $10 \%$ formalin and potassium triiodide (I2KI/Lugol's iodine) to preserve the architecture and exacerbate contrast of the specimen, and fragments of these specimens were also evaluated [15].

Each specimen was removed from the staining solution, rinsed with water to remove excess stain solution and prevent surface saturation, and dried with paper tissue. The specimens were fixed on Styrofoam to support the specimen, ensuring mechanical stability and avoiding motion artifacts during the X-ray examination. After the micro-CT study, to prevent specimen degradation and remove the stain of the iodine solution, the specimens were returned to $10 \%$ formalin solution for 7 days and followed for traditional histological analysis. 


\section{Micro-CT and image analysis}

Two systems were systems were used to acquire the FT images. The SkyScan 1173 (version 1.6.9.4; Bruker micro-CT, Kontich, Belgium) was used to acquire the group of twelve specimens. GE Phoenix was used to acquire images of three specimen and fragments of FT. X-ray energies, currents, and exposure times were adapted individually to optimize image acquisition for each specimen. Energy ranged from 40 to $130 \mathrm{kV}$, current from 61 to $200 \mu \mathrm{A}$, exposure time from 250 to $100 \mathrm{~ms}$, scan duration between 13 and $88 \mathrm{~min}$, and voxel size between 9.26 and $39.45 \mu \mathrm{m}$. Post-processing analysis and image reconstructions were performed using the Bruker micro-CT (version 1.16; Kontich, Belgium) and DataViewer (version 1.5.6.2 64 bit 2017; Kontich, Belgium).

\section{Results}

In total, 15 FT specimens were examined from 14 patients who agreed to participate in the study (age: $26-42$ years; median: 34 years). The following FT segments were examined: the intramural $(n=6)$, isthmus $(n=14)$, and infundibulum and fimbriae $(n=15)$. The iodine uptake in these specimens was high, the tissues were well defined, and contrast was sufficient for morphological analysis of the FT. The microtomographic analysis was sufficient for microscopic examination, with different contrasts used in the tissues (Figure 1). The morphology of the FT mucosa was examined and recorded on the basis of the topography and anatomical characteristics previously described. The FT was categorized according to the anatomy as follows: intramural or interstitial portion; isthmic portion; ampullary portion; and infundibular portion and fimbria (Figure S1).

\section{FT mucosa: intramural segment and transition to the isthmus}

The uterine segment of the FT begins in the ostium, which is symmetrical and rounded. In its trajectory, mu- cosal folds appeared as the FT mucosa developed laterally. A single fold was observed near the ostium (Figure 2A, B). More folds (not more than five) appeared laterally near the extrauterine segment. The mucosal folds were complex and formed crypts along this segment (Figure 2C, D). The length of these folds did not reach $50 \%$ of the diameter of the FT lumen, corresponding to a few tenths of a millimeter. The overlap of folds created blind ends (Figures S2 and S3). The analysis of coronal sections of the lumen indicated that the folds were bulkier and distended the lumen depending on their size (Figure S4). The lumen suddenly became dilated, and the mucosal folds became more numerous, occupying more space in the lumen. With increasing distance from the uterine cavity, the folds became thinner and more complex although their number increased only slightly (Figure S5).

\section{Isthmic mucosa and transition to the ampulla}

The folds in this segment were comparatively more abundant and longer than those in the intramural segment but smaller than the FT lumen. In addition, these folds were not related to those located anteriorly and did not originate from them (Figures S6, S7, and S8). The tortuosity of the isthmus was low. Tortuosity began after the uterine segment and limited the consistent serial sectioning of the mucosa and the simultaneous analysis of the specimens in three planes.

The mucosal folds with a broad base began to taper and increased in number. The diameter of the lumen increased (Figures 3 and S9).

\section{Ampullary mucosa}

The ampullary segment was the most complex, exhibiting high tortuosity compared to the other FT segments (Figure S10). Its intensity may be such that, in some specimens, the endosalpinx is perpendicular to the serosa. The mucosal folds were difficult to section serially because they were highly clustered. However, in some specimens,

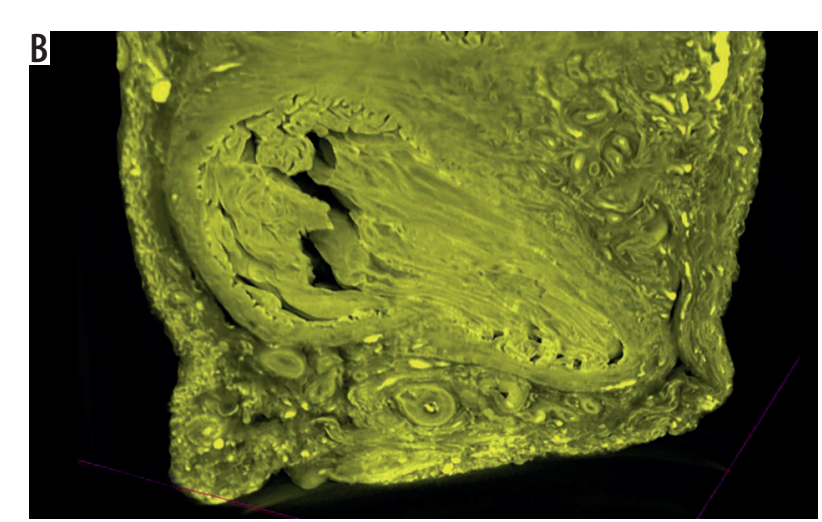

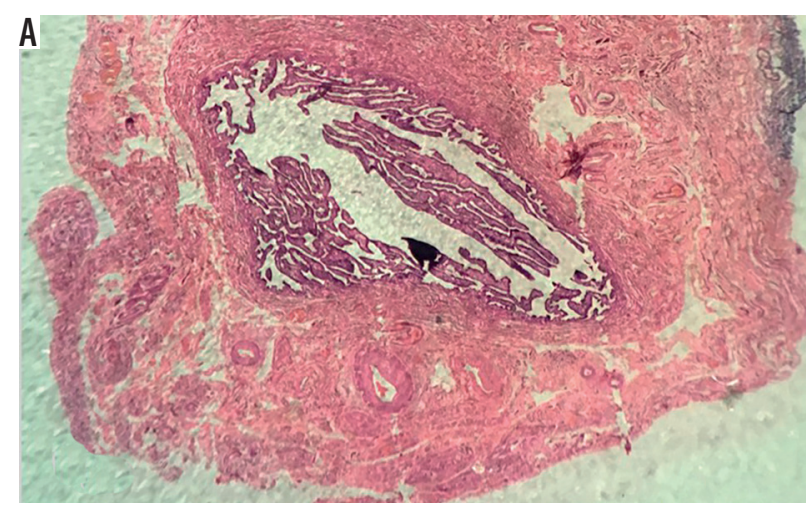

Figure 1. Fragment of the ampullary segment of the fallopian tube. A) Histological section prepared after micro-computed tomography (micro-CT) analysis. B) Three-dimensional reconstruction of the same segment based on micro-CT images 

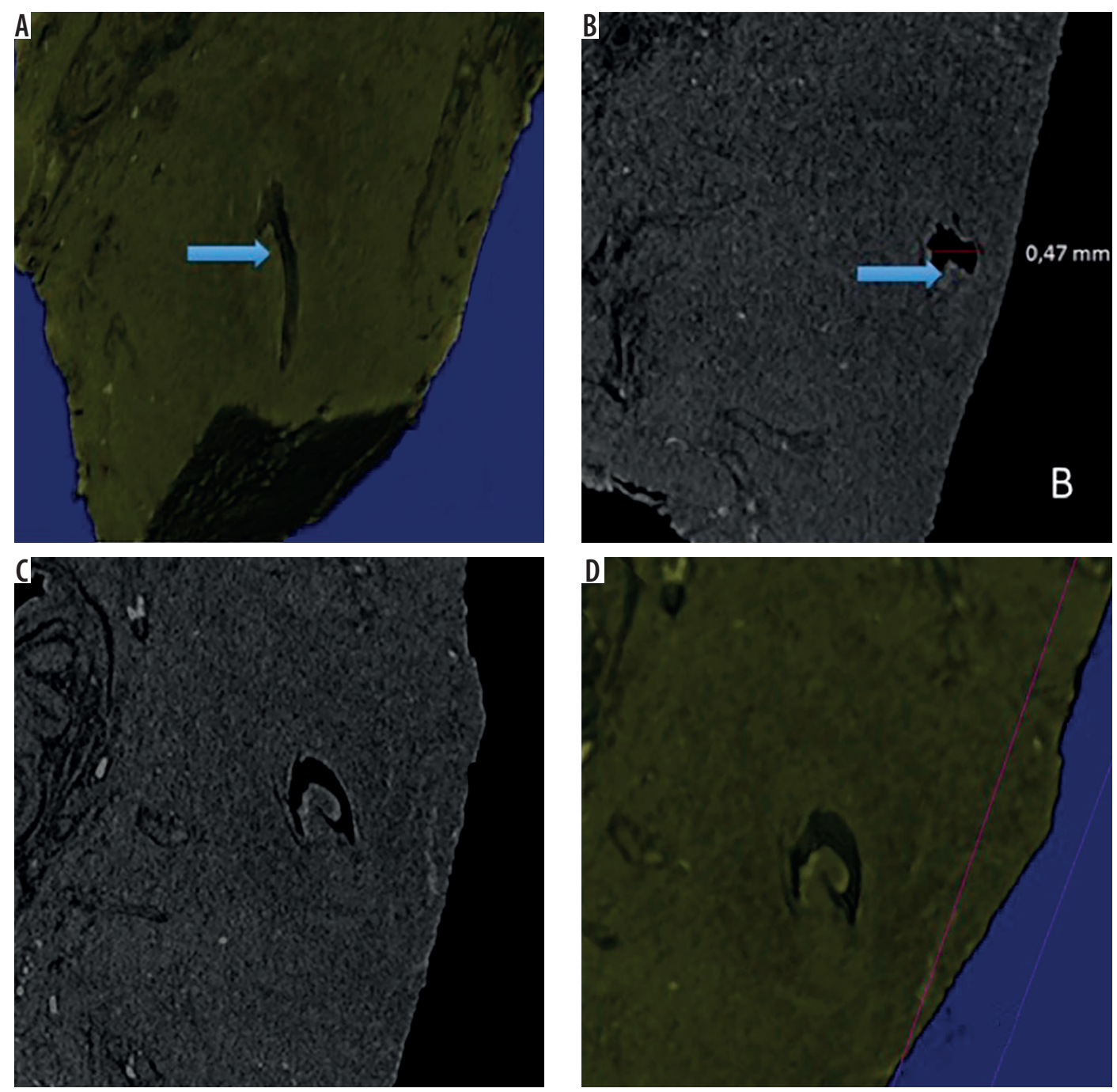

Figure 2. A) Three-dimensional reconstruction of the coronal plane of the interstitial segment near the tubal ostium (the arrow indicates the mucosal folds). B) Sagittal plane of the uterine segment of the fallopian tube near the tubal ostium. C) Sagittal plane of the fallopian tube. D) Three-dimensional reconstruction of the same segment. The mucosal folds increase in size and complexity
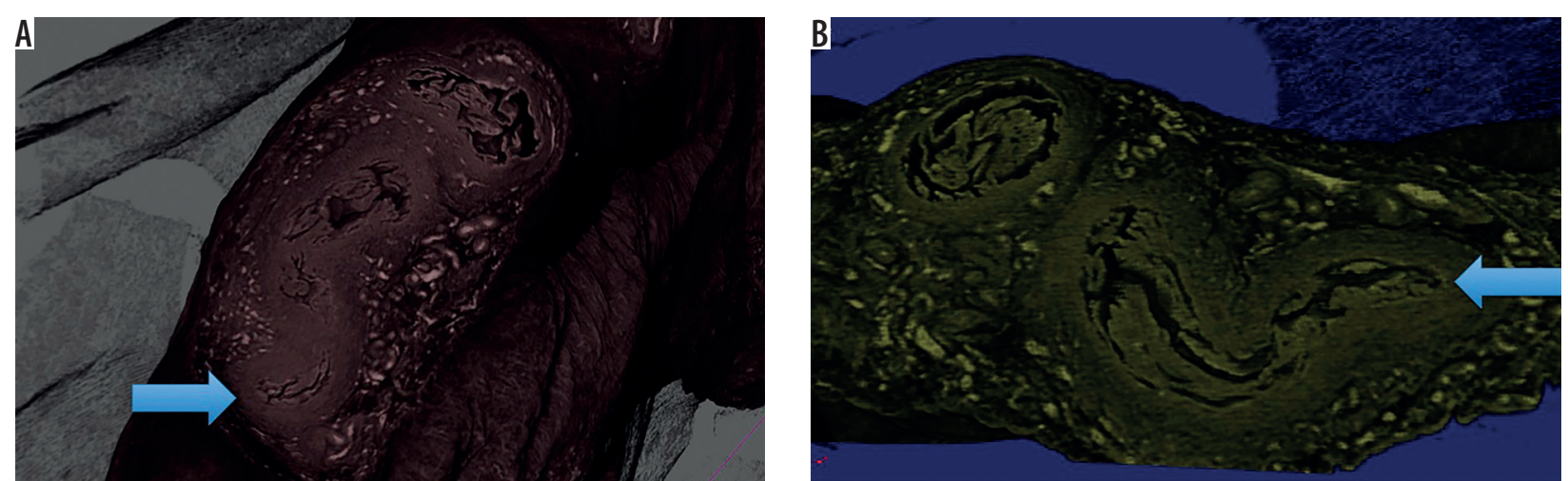

Figure 3. Three-dimensional reconstruction of the isthmus to ampulla transition. A) The mucosal folds taper abruptly and the tubal lumen becomes distended. B) Tortuosity increases unpredictably and the lumen increases in size and complexity. The arrows indicate the isthmus

the folds were more abundant in the central region of the segment, and the number of folds on the luminal surfaces closer to the periphery was smaller than that in the central region. The folds formed thin, clustered, and unpredictable branches along the entire mucosa.
The number of folds on the luminal surface was small (Figure 4) and were concentrated in the middle of the FT. The distended lumen limited three-dimensional reconstructions because a cleavage plane could not be obtained without sectioning the contralateral folds. 


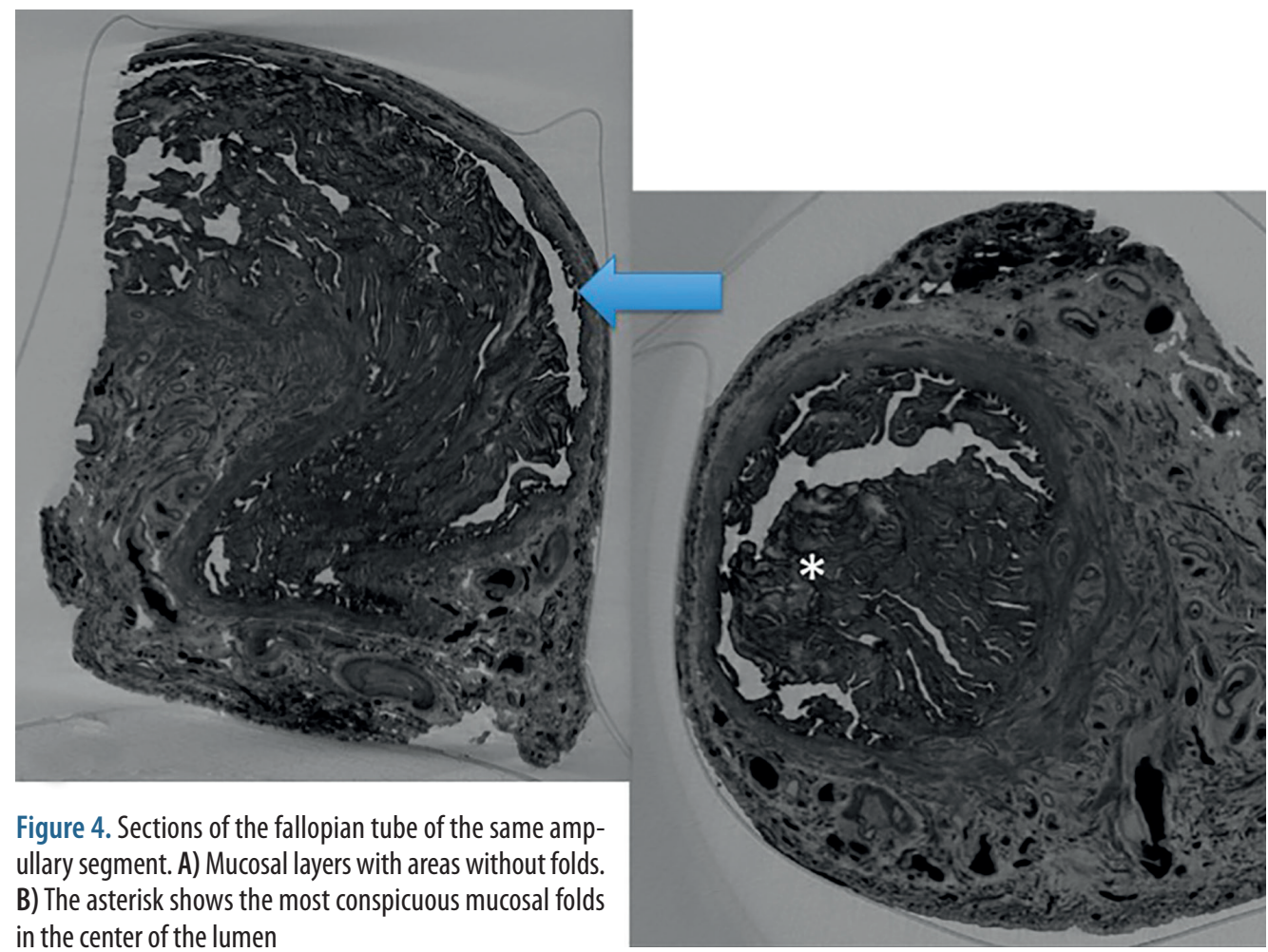

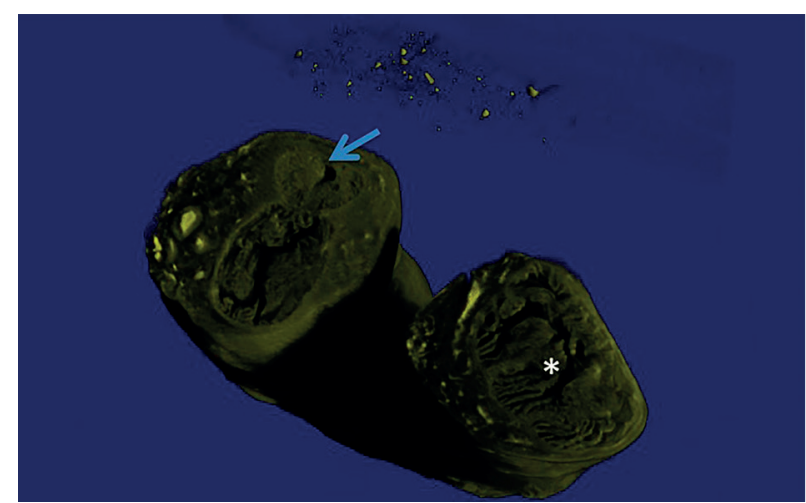

Figure 5. Three-dimensional reconstruction of tubal mucosa. The arrow shows the mucosa in the region between the isthmus and ampulla. The asterisk marks the ampullary mucosa. Note the difference between the luminal caliber and the muscle layer thickness

\section{Ampulla to infundibulum transition, fimbria, and fimbrial mucosa}

Important features were observed in the segment between the ampulla and fimbria. There was a harmonic decrease in the tortuosity that is typical of the ampulla, with apparent disorganization of the folds, and the folds formed a pleated tube toward the ostium (Figure 5). The size of the plicae decreased progressively toward the ostium in the fimbria. The mucosal folds gradually became less abundant and were anatomically similar to plicae. A reduction in plication was one of the most relevant findings in these specimens. The mucosa occupied most of the ostium, and only by longitudinally sectioning this segment was it possible to see how thin the mucosal folds were (Figure S11).

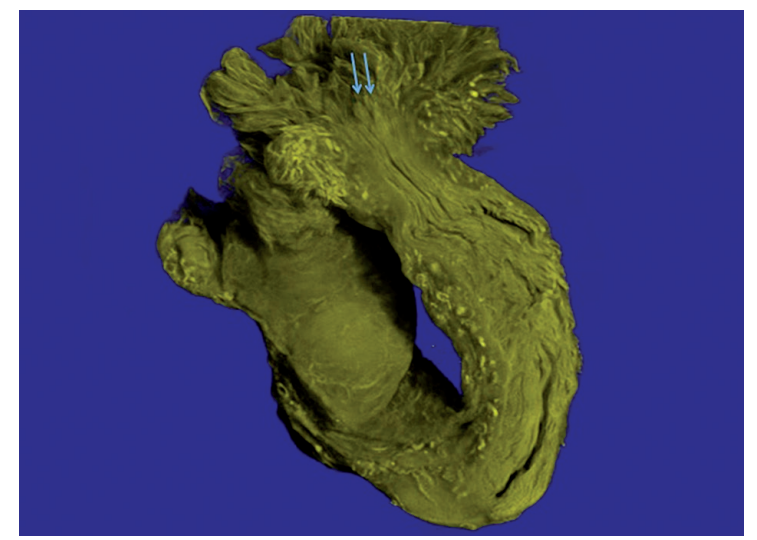

Figure 6. Three-dimensional reconstruction of the fimbria and sectioned abdominal ostium. The fimbrial mucosa is exposed and becomes narrower in the tubal ostium. Mucosal folds are present until the margins of the organ (arrows)

The muscular layer of the intramural segment caused a narrowing of the lumen near the ostium. This narrowing reduced the mucosal surface, and the influence of perimucosal tissues in narrowing was evident (Figure S12).

Furthermore, mucosal plicae closed the lumen and covered the fimbria (Figure 6) because the technique did not allow determining the limits between the serosa and the mucosa. However, there was no relationship between the intraluminal endosalpinx and the endosalpinx of the tubal fringes.

\section{Discussion}

In the present study, the microanatomy of different anatomical segments of the human endosalpinx was graphi- 
cally represented as close as possible to its in vivo state and without the interference of intraluminal contrast injection pressure, which may change the delicate morphology of the endosalpinx, its unpredictable tortuosity, and mucosal folds.

There are limited studies on the physiology of the FT due to the development of in vitro fertilization. However, the elucidation of critical functions of the FT in embryonic development and the relationship between FT and ovarian cancer have opened new perspectives on the subject [1]. The FT, particularly the tubal mucosa, participates directly in important reproductive processes related to the embryonic development by the direct contact with the epithelium, gamete maturation, rheotaxis (sperm guidance), and tubal fluid formation [16]. The tubal fluid has specific functions in protecting gametes from oxidative damage, contributing to sperm guidance, increasing viscosity to facilitate gamete and embryo mobility, promoting embryonic development, and protecting against oxidative agents $[17,18]$.

In the 1960s, it was believed that the intramural segment functions were the prevention of retrograde menstruation and postpartum reflux, the transport of the fertilized ovum, and possibly the direction of the ovum for its implantation and the transport of spermatozoa. The endosalpinx is surrounded by myometrium, and the myometrium is under hormonal control. A previous study reported an endosalpinx without mucosal folds, but this observation may be because of the limitations in histological techniques [19]. Similar to the results of the present study, a previous study describing the shape of the mucosa of the uterine segment showed the mucosa folded toward its base as tongue-like processes [20]. These folds form crypts at the beginning of the lumen and are easily observed in $3 \mathrm{D}$ reconstructions. The influence of these folds on the flow of the tubal fluid and on the motility and storage of gametes and the fertilized egg needs to be determined in humans; however, the segments of the FT mucosa that serve as sperm reservoirs appear as bulbous pockets or crypts [21]. There is evidence that spermatozoa are maintained in these pockets as a result of suppressed flagellar activity [22]. In animals without evidence of the presence of sperm reservoirs, gametes are stored by binding of the endosalpinx surface [23]. This binding occurs primarily in pockets formed by the mucosal folds. In addition to prolonging fertility, this mechanism decreases the chance of polyspermic fertilization because sperm cells are gradually released [24].

Sperm release and consequent loss of binding of sperm cells to the endosalpinx are associated with sperm capacitation [25]. Sperm capacitation leads to sperm hyperactivation, which increases the amplitude of flagellar movements on one side of the flagellum, thereby increasing motility as well as the ability to detach from the endosalpinx [26]. Hyperactivity is due to the activation of cation channels (CatSper) on the flagella. The association between the endosalpinx and sperm cells has been determined in humans; however, CatSper is rapidly activated by progesterone and pregnenolone sulfate of the tubal fluid in humans [27]. Moreover, sperm transport occurs more easily in the luteal phase, ipsilateral to the dominant follicle [28]. The anatomical segment primarily involved in hyperactivation and differences in sperm motility has not yet been determined in humans [29].

A unique characteristic of the isthmus and intramural segments is that sperm cells are transported to the abdominal ostium and the fertilized egg is transported in the direction opposite to the uterine cavity. Some studies have reported that the function of the isthmus is to select spermatozoa. Similar to the uterine cervix, the isthmus and intramural segments reduce the number of sperm cells recovered distally because the size of normal sperm cells in the ampulla is proportionally greater than that in the ejaculate [30]. The mechanical obstruction offered by the isthmus to the ascent in the distal portion of the FT also protects against the migration of pathogens. This protection has also been related to the presence of mucus in the isthmus; however, the effect of the isthmic mucosa on these functions needs to be determined. To the best of our knowledge, to date, no study has evaluated the effects of sudden occurrence and interruption of folds and of the lack of continuity between the folds on the dynamics of the tubal fluid.

The transition between the isthmus and ampulla is marked by unique characteristics, including greater tortuosity of the FT and increased number of mucosal folds and increased lumen. Some authors state that the human FT is not coiled, in contrast to that in other mammals wherein the entire FT, including the serosa, is coiled [1]. In humans, the tubal serosa is symmetrical, with high tortuosity of the mucosa and lumen. The decrease in or absence of tortuosity is related to infertility in some animal models and pathological conditions in humans [31,32]. Other studies have suggested that tortuosity is related to infertility [33]. In the present study, specimens were collected from patients with previous pregnancies; however, the unpredictability and variations among the specimens limited quantification of the mucosal tortuosity.

The present study revealed larger and bulkier mucosal folds in the central region of the FT segment, with fewer and smaller folds in the antimesenteric segment; these findings have not been described in the literature. However, in studies on tubal gestation, the antimesenteric segment is the most common point of rupture, which demonstrates the vulnerability of this region [34]. In contrast to the segments closer to the uterus, the ampullary mucosa has a larger area and has characteristics similar to a virtual cavity, as described previously [35]. However, this finding cannot be confirmed because the formalin solution causes tissue shrinkage.

The transition from the ampulla to the infundibulum is abrupt, and the mucosa exhibits marked changes. 
The mucosal folds decrease in size and number, become harmonic, and their diameter is progressively reduced toward the abdominal tubal ostium. The number of folds in the ostium varies, and the overlap of mucosal folds may affect this morphology. A previous study has shown that the abdominal ostium has 10-12 folds [36]. The abdominal ostium has a narrower lumen and prevents infections, giving a sausage-like appearance to FTs in pathological conditions.

The tubal mucosa progresses toward the fimbria beyond the ostium, becomes enlarged, and exhibits asymmetrical and irregularly shaped folds. Three-dimensional reconstructions show the morphology of folds but cannot precisely determine the limit between the uterine mucosa and serosa. No other radiological method shows the fimbriae and their typical microscopic appearance. The margins of the endosalpinx have been recorded graphically for more than 100 years using different methods, including X-ray, ultrasonography, scintigraphy, and MRI [7-9]. The need to fill the tubal lumen with contrast media and the low image resolution limit the identification of the actual FT morphology by traditional radiological methods. Similarly, although electron microscopy has helped to produce three-dimensional images of the human endosalpinx for the past 50 years, it is only in small segments [37]. Recently, optical coherence tomography allowed three-dimensional reconstructions with good depth and the precise identification of several anatomical structures, including muscle, mucosal, and serous layers, and allowed in vivo studies of the isthmus [38].

Micro-CT is a non-destructive method used for the microscopic examination of biological tissues. Created for industrial use, it was first used to analyze human bone fragments. The use of contrast media in soft biological tis- sues has considerably increased the utility of the method, and the results can add new information to traditional histological methods [39]. Micro-CT is widely used in human tissue research and can be applied in the preoperative assessment of breast cancer, post-mortem virtual studies of fetuses and embryos, and medical training $[15,40]$.

The menstrual cycle is an important variable in studying the microanatomy of the reproductive tract. In the present study, this variable was not considered because many patients were under hormonal treatment for abnormal uterine bleeding. However, changes in the endosalpinx related to the menstrual cycle included the ratio of ciliated to secretory cells and the number of cilia on the tubal surface. Nonetheless, to the best of our knowledge, no changes in the microscopic architecture of the endosalpinx have been described to date [41].

Our results indicate that the microscopic morphology and anatomy of the endosalpinx can be evaluated and reconstructed in $3 \mathrm{D}$ using micro-CT. This technique adequately identified mucosal folds in the uterine segment, the sudden appearance of mucosal folds in the isthmus, the presence of folds primarily in the mesenteric segment of the endosalpinx in the ampulla, and the tubal ostium. Therefore, micro-CT is essential for the microscopic study of the anatomy of the reproductive tract. Further studies are necessary to elucidate the physiology and determine the effect of the microanatomy of the FT mucosa on the dynamics of the tubal fluid and gamete transport.

\section{Conflict of interest}

The authors report no conflict of interest.

\section{References}

1. Li S, Winuthayanon W. Oviduct: roles in fertilization and early embryo development. J Endocrinol 2017; 232: R1-R26.

2. Hunter RHF. The Fallopian tubes. Their role in fertility and infertility. $1^{\text {st }}$ ed. Springer-Verlag, Berlin Heidelberg 1988.

3. Yaniz JL, Lopez-Gatius F, Hunter RH. Scanning electron microscopic study of the functional anatomy of the porcine oviductal mucosa. Anat Histol Embryol 2006; 35: 28-34.

4. Nayak RK, Ellington EF. Ultrastructural and ultracytochemical cyclic changes in the bovine uterine tube (oviduct) epithelium. Am J Vet Res 1977; 38: 157-168.

5. Odor DL, Gaddum-Rosse P, Rumery RE, Blandau RJ. Cyclic variations in the oviuductal ciliated cells during the menstrual cycle and after estrogen treatment in the pig-tailed monkey, Macaca nemestrina. Anat Rec 1980; 198: 35-57

6. Yániz JL, Carretero T, Recreo P, et al. Three-dimensional architecture of the ovine oviductal mucosa. Anat Histol Embryol 2014; 43: 331-340.

7. Rubin IC. Uterotubal insufflation. CV Mosby Co, St. Louis 1947.
8. Deichert U, Schleif R, van de Sandt M, Juhnke I. Transvaginal hysterosalpingo-contrast-sonography (Hy-Co-Sy) compared with conventional tubal diagnostics. Hum Reprod 1989; 4: 418-424.

9. Carrascosa P, Capuñay C, Vallejos J, et al. Two-dimensional and three-dimensional imaging of uterus and fallopian tubes in female infertility. Fertil Steril 2016; 105: 1403-1420.e7.

10. Dickinson ME. Multimodal imaging of mouse development: tools for the postgenomic era. Dev Dyn 2006; 235: 2386-2400.

11. Mizutani R, Takeuchi A, Uesugi K, et al. Microtomographic analysis of neuronal circuits of human brain. Cereb Cortex 2010; 20: 1739-1748.

12. Metscher BD. MicroCT for comparative morphology: simple staining methods allow high-contrast 3D imaging of diverse non-mineralized animal tissues. BMC Physiol 2009; 9: 11.

13. Werner M, Chott A, Fabiano A, Battifora H. Effect of formalin tissue fixation and processing on immunohistochemistry. Am J Surg Pathol 2000; 24: 1016-1019.

14. Castro PT, Matos APP, Aranda OL, et al. Tuboperitoneal fistula, ectopic pregnancy, and remnants of fallopian tube: a confocal micro- 
tomography analysis and 3D reconstruction of human fallopian tube pathologies. J Matern Fetal Neonatal Med 2019; 32: 3082-3087.

15. Hutchinson JC, Kang X, Shelmerdine SC, et al. Postmortem microfocus computed tomography for early gestation fetuses: a validation study against conventional autopsy. Am J Obstet Gynecol 2018; 218 : 445.e1-445.e12.

16. Morales P, Palma V, Salgado AM, Villalon M. Sperm interaction with human oviductal cells in vitro. Hum Reprod 1996; 11: 1504-1509.

17. Georgiou AS, Sostaric E, Wong CH, et al. Gametes alter the oviductal secretory proteome. Mol Cell Proteomics 2005; 4: 1785-1796.

18. Oren-Benaroya R, Orvieto R, Gakamsky A, et al. The sperm chemoattractant secreted from human cumulus cells is progesterone. Hum Reprod 2008; 23: 2339-2345.

19. Beck LR, Boots LR. The comparative anatomy, histology and morphology of the mammalian oviduct. In: Johnson AD, Foley CW (eds.). The oviduct and its functions. Academic Press, New York London 1974; 14-16.

20. Rocca M, el Habashy M, Nayel S, Madwar A. The intramural segment and the uterotubal junction: an anatomic and histologic study. Int J Gynaecol Obstet 1989; 28: 343-349.

21. Bedford JM, Mock OB, Nagdas SK, et al. Reproductive features of the eastern mole (Scalopus aquaticus) and star-nose mole (Condylura cristata). J Reprod Fertil 1999; 117: 345-353.

22. Bedford JM, Breed WG. Regulated storage and subsequent transformation of spermatozoa in the fallopian tubes of an Australian marsupial, Sminthopsis crassicaudata. Biol Reprod 1994; 50: 845-854.

23. Hunter RH. Sperm transport and reservoirs in the pig oviduct in relation to the time of ovulation. J Reprod Fertil 1981; 63: 109-117.

24. Suarez SS. Regulation of sperm storage and movement in the mammalian oviduct. Int J Dev Biol 2008; 52: 455-462.

25. Ignotz GG, Cho MY, Suarez SS. Annexins are candidate oviductal receptors for bovine sperm surface proteins and thus may serve to hold bovine sperm in the oviductal reservoir. Biol Reprod 2007; 77: 906-913.

26. Demott RP, Suarez SS. Hyperactivated sperm progress in the mouse oviduct. Biol Reprod 1992; 46: 779-785.

27. Lishko PV, Botchkina IL, Kirichok Y. Progesterone activates the principal Ca2+ channel of human sperm. Nature 2011; 471: 387-391.

28. Kunz G, Beil D, Deininger $H$, et al. The dynamics of rapid sperm transport through the female genital tract: evidence from vaginal sonography of uterine peristalsis and hysterosalpingoscintigraphy. Hum Reprod 1996; 11: 627-632.

29. Ardon F, Markello RD, Hu L, et al. Dynamics of bovine sperm interaction with epithelium differ between oviductal isthmus and ampulla. Biol Reprod 2016; 95: 90.

30. Pauerstein CJ. Clinical implications of oviductal physiology and biochemistry. Gynecol Invest 1975; 6: 253-264.

31. Prunskaite-Hyyryläinen R, Skovorodkin I, Xu Q, et al. Wnt4 coordinates directional cell migration and extension of the Müllerian duct essential for ontogenesis of the female reproductive tract. Hum Mol Genet 2016; 25: 1059-1073.

32. Ferguson L, Kaftanovskaya EM, Manresa C, et al. Constitutive notch signaling causes abnormal development of the oviducts, abnormal angiogenesis, and cyst formation in mouse female reproductive tract. Biol Reprod 2016; 94: 67.

33. Hwang TS, Song J. Morphometrical changes of the human uterine tubes according to aging and menstrual cycle. Ann Anat 2004; 186: 263-269.

34. Stock RJ. Histopathologic changes in tubal pregnancy. J Reprod Med 1985; 30: 923-928.

35. Ranney B, Leonardson G. Volume reduction of the uterus during and soon after hysterectomy. Am J Obstet Gynecol 1986; 155: 354-357.

36. Stange HH. Functional morphology of the fimbrial end of the human tube and the epoophoron. Arch Gynakol 1952; 182: 77-103.

37. Clyman MJ. Electron microscopy of the human fallopian tube. Fertil Steril 1966; 17: 281-301.

38. Kirillin M, Panteleeva O, Yunusova E, et al. Criteria for pathology recognition in optical coherence tomography of fallopian tubes. J Biomed Opt 2012; 17: 081413-1.

39. Metscher BD. MicroCT for developmental biology: a versatile tool for high-contrast 3D imaging at histological resolutions. Dev Dyn 2009; 238: 632-640.

40. Tang R, Saksena M, Coopey SB, et al. Intraoperative micro-computed tomography (micro-CT): a novel method for determination of primary tumour dimensions in breast cancer specimens. Br J Radiol 2016; 89: 20150581.

41. Patek E. The epithelium of the human Fallopian tube. A surface ultrastructural and cytochemical study. Acta Obstet Gynecol Scand Suppl 1974; 31: 1-28. 